\title{
ON OBTAINING NUMERICAL SOLUTIONS FOR SOME REACTING FLOW PROBLEMS BY USING DIFFERENT MATLAB TOOLS
}

\author{
Maksims Marinaki \\ Institute of Mathematics and Computer Science, University of Latvia, Latvia \\ maksims.marinaki@lu.lv
}

\begin{abstract}
The author considers the reacting flow problem modelling and simulation possibilities by using the Matlab software and its packages. These types of problems naturally arise when one wishes to mathematically describe the processes of combustion or gasification of different fuels including biomass. These topics have been widely researched by different institutes in Latvia during the last years. The Matlab software and its packages are widely considered as a very powerful tool when it comes to matrix operations. Such operations are naturally involved when it comes to discretizing the systems of partial differential equations. It is possible to use the PDE tools package, FEATool package or create the author's own code in Matlab for tackling the types of problems, such as obtaining stationary or non-stationary solutions for mass fraction and temperature equations, describing the reacting flow. First, the model problem is proposed - the one, where we take a list of chemical reactions and construct the source terms for mass fraction and temperature equations. These models are parameterized and thus we briefly discuss the parametrization aspects as well. Afterwards, for different types of problems, such as obtaining solutions for stationary or non-stationary equations, serving as models of diffusion flames, the author obtains the simulation results, temperature and mass fraction distributions and analyses the advantages and disadvantages of each tool.
\end{abstract}

Keywords: modelling, simulation, combustion, PDEs, Matlab.

\section{Introduction}

The main goal of the current research refers to the EU 2030 targets to reduce overall greenhouse gas (GHG) emissions by $40 \%$, to increase the energy production efficiency and the utilization of the renewable energy sources to at least $32 \%$ share, thus minimizing the effect of heat producers on the GHG emissions and global warming. In this context, the use of different agriculture and harvesting residues (wood, wheat straw, rape straw) for energy production is crucial [1]. The use of biofuels for energy production nowadays reaches already $14-15 \%$ of the world's total energy consumption and has already provided a significant reduction of greenhouse gas emissions, more efficient use of biofuels, such as straw [2].

These challenges led to appearance of several research collaboration projects in Latvia held in the last decade. In [3; 4] amongst many other articles, there has been a collaboration between two research institutes: the Institute of Mathematics and Computer Science of the University of Latvia and the Institute of Physics of the University of Latvia. The researchers have been widely interested in topics such as an efficient combustion of biomass for a very long time and have facilitating the experiments involving the combustion and gasification of different fuels.

Regarding the mathematical modelling part there has been a necessity of formulating the differential problems, setting initial and boundary conditions and choosing reactivity parameters wisely, so that the modelling results are close to the experimental results to a certain extent. In [5] the modern theory and applications of combustion and gasification processes are introduced. More classical theory is found in [6] and [7]. The computer simulations have been performed by means of different Matlab tools. Matlab is considered as a very useful tool when it comes to matrix operations. The description of its built-in features can be found in [8-10]. The description of the additional package FeaTool is found in [11].

What we present here is the brief review of the experimental results from articles such as $[3 ; 4]$, some recent modelling results and the discussion of modelling tools used and their usage possibilities for further research. In this particular talk the author wants to summarize his part in the aforementioned activities and discuss the advantages and possibilities of usage of the developed framework. This discussion might be useful for researchers, participating in new related projects naturally appearing today.

\section{Materials and methods}

Here we provide a brief review of the experimental results, published in prior works, such as $[3,4]$. In order to initiate the discussion on comparison of experimental and modelling (to be presented below) 
results, the pictures from the mentioned articles the author has been involved in have been replicated in the present article.

The experimental setup composed of a biomass gasifier and a combustor is presented in Fig. 1.

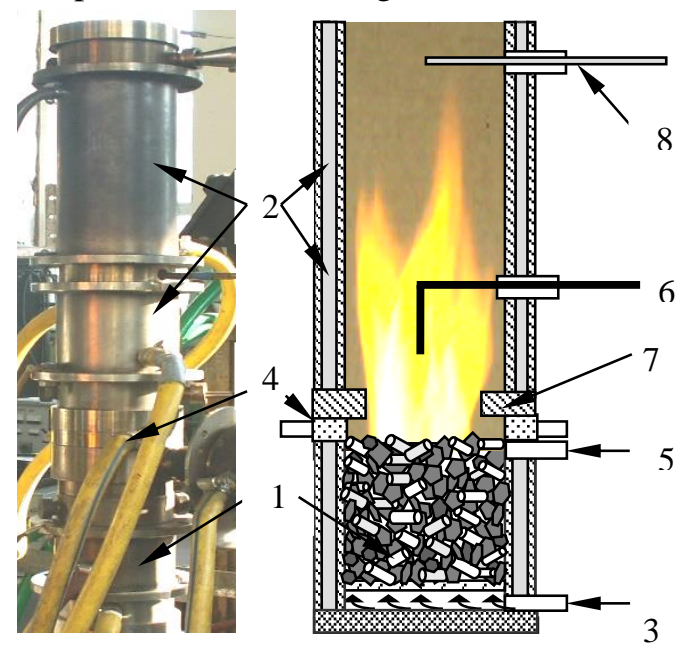

Fig. 1. Digital image of computational domain (left) and schematic of experimental device (right), different parts of the experimental device: 1 - gasifier with a biomass mixture; 2 - cooling jacket of the combustor; 3 - primary air supply; 4 - secondary air supply; 5 - propane flame injection; 6 - positively biased electrode; 7 - annular inlet nozzle of the combustor; 8 - diagnostic section.

In the following Figs. 2 and 3 the experimental results on the impact of the electrical field or propane supply on $\mathrm{CO}_{2}$ volume fraction and temperature distributions have been obtained in the published works discussed above. Different types of fuel are considered. Amongst these there have been experiments including different biomass mixtures and effects of the magnetic field and published collaboration results where the author of the present talk has been involved with. For the sake of non-excessive selfcitation the links are not provided here, but can be found in Erdev journal archives.
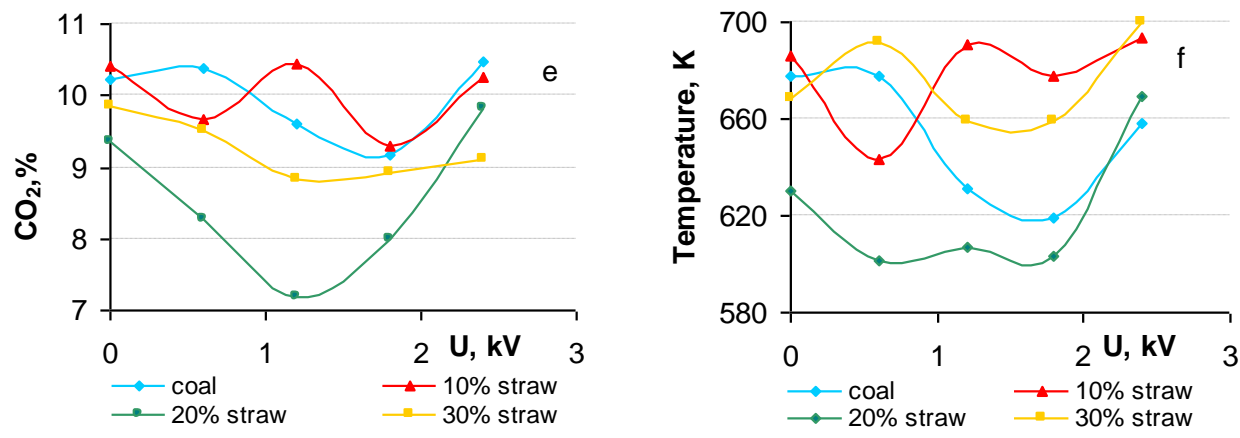

Fig. 2. Electric field-induced variations of $\mathrm{CO}_{2}$ volume fraction (left) and product temperature (right)

The temperature and mass fraction distributions can be obtained in greater detail by modelling of chemical reactions by considering the reaction mechanism and the systems of PDEs. After solving the system numerically, the dependence graphs, such as the ones above, can be obtained. Depending on parametrization and the initial/boundary conditions we can obtain closeness to the experimental data to a certain extent. Some data can be entered straightforwardly in dimensional form of the equations in the following section. The modelling of reactions, however, is the tricky part, since hundreds of reactions are usually modelled by a mechanism consisting of several only. The reactivity parameters in the models in the following section therefore are usually left for modellers to vary.

The peculiarities of the mathematical modelling and the concept of comparing the modelling results with the experimental data to be explained in the next sections have been used in many published works, such as $[3 ; 4]$. The author of the present talk has been involved in them taking part in providing the 
modelling and simulation stage - developing model in PDE system form and using Matlab computer software to obtain its solution.
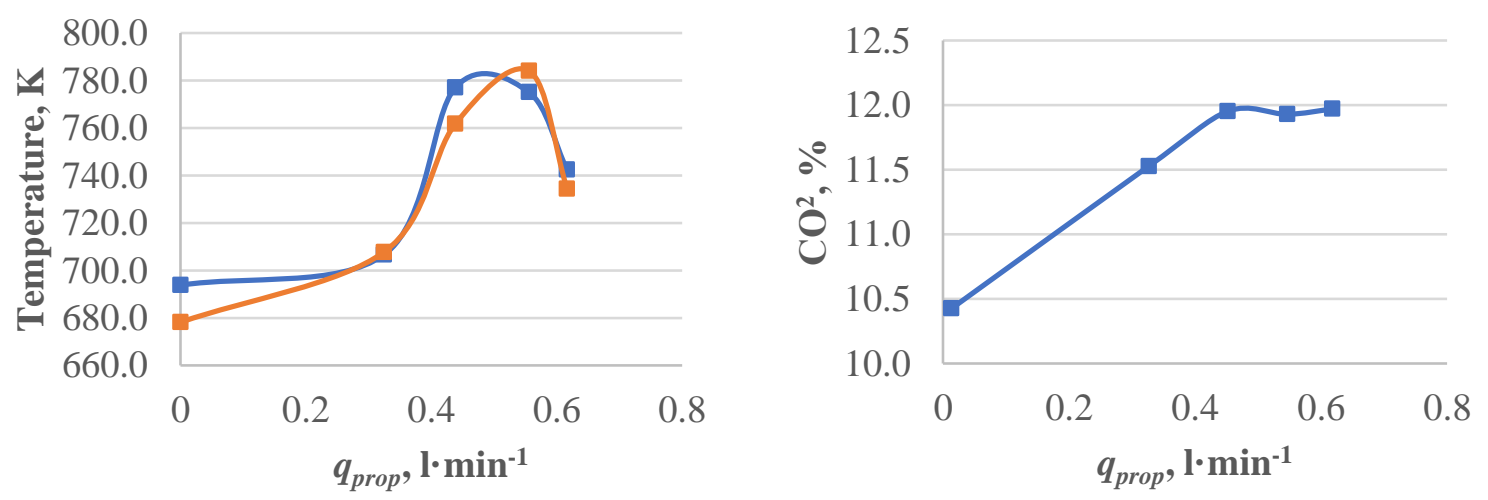

Fig. 3. Effect of propane supply on average values of the flame:

temperature (left) and volume fraction of $\mathrm{CO}_{2}$ versus injection of propane (right)

\section{Mathematical Modelling}

Here we present the PDE systems, and according to abstract discuss the Matlab different tools and the parametrization. The latter is the key to the comparison results.

The model with three combustion reactions are considered:

$$
\begin{aligned}
& \mathrm{O}_{2}+\mathrm{CO} \rightarrow \mathrm{O}+\mathrm{CO}_{2}, \\
& \mathrm{CO}_{2}+\mathrm{H} \rightarrow \mathrm{CO}+\mathrm{OH}, \\
& \mathrm{H}_{2}+\mathrm{OH} \rightarrow \mathrm{H}_{2} \mathrm{O}+\mathrm{H} .
\end{aligned}
$$

Amongst the other mechanisms used in publications such as [3;4], this one is chosen for the present illustration, since these particular species are usually the ones sought by the team facilitating the experiments.

The reaction mechanism (1) leads to 9 PDEs ( 8 species equations and one temperature equation).

The species equation for the specie $Y_{k}$ has its form [5-7]:

$$
\rho \frac{D Y_{k}}{D t}=\nabla \cdot\left(\rho D \nabla Y_{k}\right)+\dot{w}_{k} .
$$

The term $\dot{\omega}_{k}$ is the sum over all reactions:

$$
\sum_{j=1}^{M} \dot{\omega}_{k j}
$$

where $\dot{\omega}_{k j}$-expressed in form $\dot{\omega}_{k j}=q_{j} w_{k} v_{k j}$.

The explanation of this form starts with the $v_{k j}$ multiplier. This is constructed as following: we consider $M$ reactions in our model, through which $N$ species are reacting. We consider forward reactions here and each $j$-th reaction, $j=1 . . M$, usually has the form

$$
\sum_{k=1}^{N} v_{k j}{ }^{\prime} M_{k} \rightarrow \sum_{k=1}^{N} v_{k j}{ }^{\prime \prime} M_{k}, j=1 . . M
$$

where $v_{k j}$-number of moles of the $k$-th specie in the $j$-th reaction;

$M_{k}$ - nomenclature of the corresponding specie.

Then we denote $v_{k j}$ : $=v^{\prime \prime}{ }_{k j}-v^{\prime}{ }_{k j}$. So we have only one stoichiometric coefficient describing the reaction instead of two, which can be either positive or negative. The $w_{k}$ is the molecular weight of specie $k$, while $q_{j}$ is the multiplier that covers the Arrhenius law $[5 ; 6]$.

The Arrhenius law lies in the core of the reaction term. For the forward reaction it has the following form: 


$$
\begin{gathered}
q_{j}=K_{j} \prod_{k=1}^{N} N_{k}^{v_{k j^{\prime}}}, \\
\mathrm{K}_{\mathrm{j}}=\mathrm{A}_{\mathrm{j}} \mathrm{e}^{\frac{-\mathrm{E}_{\mathrm{j}}}{\mathrm{RT}} \mathrm{T}^{\beta_{\mathrm{j}}} .}
\end{gathered}
$$

Here $N_{k}$ is the concentration of the specie $k$ as the amount of moles per cubic meter. The reactivity parameters for the reaction $j$ are the pre-exponential factor $A_{j}$, the activation energy $E_{j}$ and the temperature exponent $\beta_{j}$ and are either to be determined from the existing tables or their values would be estimated. $R$ is the ideal gas constant.

The concentration - mass fraction relation to use is

$$
N_{k}=\frac{\rho Y_{k}}{w_{k}} .
$$

The numeration for the species is as follows:

$$
\begin{gathered}
Y_{1}:=Y\left(\mathrm{O}_{2}\right), Y_{2}:=Y(\mathrm{CO}), Y_{3}:=Y(0), Y_{4}:=Y\left(\mathrm{CO}_{2}\right), Y_{5}:=Y(\mathrm{H}), \\
Y_{6}:=Y(\mathrm{OH}), Y_{7}:=Y\left(\mathrm{H}_{2}\right), Y_{8}:=Y\left(\mathrm{H}_{2} \mathrm{O}\right) .
\end{gathered}
$$

Then the matrix $v$ of the stoichiometric coefficients (4) in the reaction mechanism (1) is

$$
\left(\begin{array}{lll}
-1 & 0 & 0 \\
-1 & 1 & 0 \\
1 & 0 & 0 \\
1 & -1 & 0 \\
0 & -1 & 1 \\
0 & 1 & -1 \\
0 & 0 & -1 \\
0 & 0 & 1
\end{array}\right) \text {, and the matrix } \dot{w} \text { is }\left(\begin{array}{lll}
-q_{1} w_{1} & 0 & 0 \\
-q_{1} w_{2} & q_{2} w_{2} & 0 \\
q_{1} w_{3} & 0 & 0 \\
q_{1} w_{4} & -q_{2} w_{4} & 0 \\
0 & -q_{2} w_{5} & q_{3} w_{5} \\
0 & q_{2} w_{6} & -q_{3} w_{6} \\
0 & 0 & -q_{3} w_{7} \\
0 & 0 & q_{3} w_{8}
\end{array}\right) \text {. }
$$

According to the Arrhenius law $(5,6)$,

$$
\begin{aligned}
& q_{1}=A_{1} e^{\frac{-E_{1}}{R T}\left(\frac{\rho Y_{1}}{w_{1}}\right)^{1}\left(\frac{\rho Y_{2}}{w_{2}}\right)^{1},} \\
& q_{2}=A_{2} e^{\frac{-E_{2}}{R T}\left(\frac{\rho Y_{4}}{w_{4}}\right)^{1}\left(\frac{\rho Y_{4}}{w_{4}}\right)^{1},} \\
& q_{3}=A_{3} e^{\frac{-E_{3}}{R T}}\left(\frac{\rho Y_{6}}{w_{7}}\right)^{1}\left(\frac{\rho Y_{6}}{w_{7}}\right)^{1} .
\end{aligned}
$$

The powers of one have been intentionally left in order to show that the same template can be used for other coefficents not necessarily equal to unity.

Also we consider the axial velocity component $w$ only. The diffusivity $D$ is considered as a constant and equal for every specie in the system. In this case, the species equations are (terms are divided by $\rho$ ):

$$
\left\{\begin{array}{l}
\frac{\partial Y_{1}}{\partial t}+w \frac{\partial Y_{1}}{\partial x}=D \Delta Y_{1}-\frac{q_{1} w_{1}}{\rho}, \\
\frac{\partial Y_{2}}{\partial t}+w \frac{\partial Y_{2}}{\partial x}=D \Delta Y_{2}+\frac{-q_{1} w_{2}+q_{2} w_{2}}{\rho}, \\
\frac{\partial Y_{3}}{\partial t}+w \frac{\partial Y_{3}}{\partial x}=D \Delta Y_{3}+\frac{q_{1} w_{3}}{\rho}, \\
\frac{\partial Y_{4}}{\partial t}+w \frac{\partial Y_{4}}{\partial x}=D \Delta Y_{4}+\frac{q_{1} w_{4}-q_{2} w_{4}}{\rho}, \\
\frac{\partial Y_{5}}{\partial t}+w \frac{\partial Y_{5}}{\partial x}=D \Delta Y_{5}+\frac{-q_{2} w_{5}+q_{3} w_{5}}{\rho}, \\
\frac{\partial Y_{6}}{\partial t}+w \frac{\partial Y_{6}}{\partial x}=D \Delta Y_{6}+\frac{q_{2} w_{6}-q_{3} w_{6}}{\rho}, \\
\frac{\partial Y_{7}}{\partial t}+w \frac{\partial Y_{7}}{\partial x}=D \Delta Y_{7}-\frac{q_{3} w_{7}}{\rho}, \\
\frac{\partial Y_{8}}{\partial t}+w \frac{\partial Y_{8}}{\partial x}=D \Delta Y_{8}+\frac{q_{3} w_{8}}{\rho} .
\end{array}\right.
$$

The temperature equation has the following form [5]: 


$$
\rho c_{p} \frac{D T}{D t}=\nabla \cdot(\lambda \nabla T)-\sum_{k=1}^{N} \Delta H_{f, k} \dot{w}_{k} .
$$

We subject the vector of unknown functions $X=\left(Y_{i}, T\right)^{T}, i=1 . .8$ to the boundary and the initial conditions:

$$
\begin{array}{r}
\left.X\right|_{\Gamma_{D}}=X_{D}, \\
\left.X\right|_{\Gamma_{N}}=0, \\
X(0)=X_{D} e^{-x},
\end{array}
$$

where $\Gamma_{D}$ - Dirichlet portion of the boundary (the inlet);

$X_{D}$ - prescribed values at $\Gamma_{D}$;

$\Gamma_{N}-$ Neumann portion of the boundary.

The smooth exponential decay initial condition is considered. In order to start the simulation one has to specify all the parameters values:

$$
\begin{gathered}
\left(\rho, c_{p}, D, \lambda, w_{i}, w, A_{j}, E_{j}, R, \triangle H_{f, i}, Y_{i, D}\right), \\
i=1 . .8, j=1 . .3 .
\end{gathered}
$$

The parameters are to be taken from different tables [12-14].

For the simulation we consider the density of the mixture $\rho$ to be $1 \mathrm{~kg} \cdot \mathrm{m}^{-3}$. The heat capacity for the mixture to be $1000 \mathrm{~J} \cdot(\mathrm{kg} \cdot \mathrm{K})^{-1}$. The molecular diffusivity $D$ is $5 \cdot 10^{-5} \mathrm{~m}^{2} \cdot \mathrm{s}^{-1}$ and the thermal conductivity $\lambda$ is $5 \cdot 10^{-2} \mathrm{~J} \cdot(\mathrm{m} \cdot \mathrm{s} \cdot \mathrm{K})^{-1}$.

The atomic weights $w_{1, . .8}$ are straightforwardly to be taken from the periodic table:

$$
\begin{aligned}
& w_{1}=0.032 \mathrm{~kg} \cdot \mathrm{mol}^{-1}, w_{2}=0.028 \mathrm{~kg} \cdot \mathrm{mol}^{-1}, w_{3}=0.016 \mathrm{~kg} \cdot \mathrm{mol}^{-1}, \\
& w_{4}=0.044 \mathrm{~kg} \cdot \mathrm{mol}^{-1}, w_{5}=0.001 \mathrm{~kg} \cdot \mathrm{mol}^{-1}, w_{6}=0.017 \mathrm{~kg} \cdot \mathrm{mol}^{-1} .
\end{aligned}
$$

The constant axial velocity is set to $1 \mathrm{~m} \cdot \mathrm{s}^{-1}$.

The pre-exponential factor and the activation energy for the reactions (1) are:

$$
\begin{gathered}
A_{1}=2.5 \cdot 10^{12} \mathrm{cgs} \text { and } E_{1}=47690 \mathrm{cal} \cdot \mathrm{mol}^{-1}=47690 \cdot 4.18 \mathrm{~J} \cdot \mathrm{mol}^{-1}, \\
A_{2}=1.5 \cdot 10^{7} \mathrm{cgs} \text { and } E_{2}=-497 \mathrm{cal} \cdot \mathrm{mol}^{-1}=-497 \cdot 4.18 \mathrm{~J} \cdot \mathrm{mol}^{-1}, \\
A_{3}=2.2 \cdot 10^{12} \mathrm{cgs} \text { and } E_{3}=3430 \mathrm{cal} \cdot \mathrm{mol}^{-1}=3430 \cdot 4.18 \mathrm{~J} \cdot \mathrm{mol}^{-1} .
\end{gathered}
$$

For this model we were lucky enough to find the same reaction mechanism in [13]. But this result is an empirical one suitable for some applications and the mechanism itself might be an idealisation for different models, hence additional parameter optimization still could be considered.

The enthalpies of formation for the species are $\triangle H_{f, 1}=0 \mathrm{~J} \cdot \mathrm{mol}^{-1}, \Delta H_{f, 2}=-110435 \mathrm{~J} \cdot \mathrm{mol}^{-1}, \Delta H_{f, 3}=248918 \mathrm{~J} \cdot \mathrm{mol}^{-1}, \Delta H_{f, 4}=-393129 \mathrm{~J} \cdot \mathrm{mol}^{-1}$, $\triangle H_{f, 5}=217778 \mathrm{~J} \cdot \mathrm{mol}^{-1}, \Delta H_{f, 6}=38957 \mathrm{~J} \cdot \mathrm{mol}^{-1}, \Delta H_{f, 7}=0 \mathrm{~J} \cdot \mathrm{mol}^{-1}, \Delta H_{f, 8}=-241604 \mathrm{~J} \cdot \mathrm{mol}^{-1}[12]$.

Finally, to prescribe the constant values on the Dirichlet portion of the boundary, we use the atomic weight ratios and then solve the system for the mass fractions at stoichiometry:

$$
\left\{\begin{array}{l}
\frac{Y_{1}}{Y_{2}}=\frac{8}{7} \\
\frac{Y_{1}}{Y_{4}}=\frac{8}{11} \\
\frac{Y_{4}}{Y_{5}}=44 \\
\frac{Y_{4}}{Y_{7}}=22 \\
\frac{Y_{7}}{Y_{6}}=\frac{2}{17} \\
Y_{1}+Y_{2}+Y_{4}+Y_{5}+Y_{6}+Y_{7}=1 .
\end{array}\right.
$$


The pure products in this chain of reactions are set to zero on this portion of the boundary. After the described modelling stages, the equations in this particular differential format with all the initial and boundary conditions set are made possible to solve in Matlab. This is the topic of the next section.

\section{Results and discussion}

First, in Fig. 4 we provide the pictorial representations of the selected fields, same as considered in the experiment sections, obtained by solving $(10,11)$ in Matlab. Solution aspect to be discussed below.
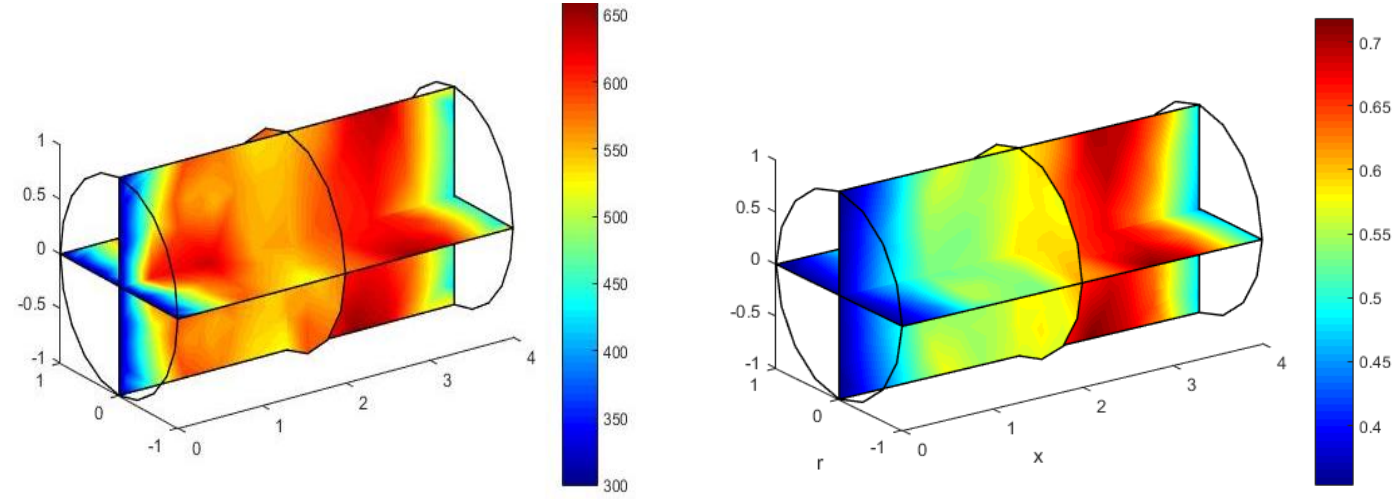

Fig. 4. Distribution of temperature (left) and $\mathrm{CO}_{2}$ mass fraction (right) in the combustion chamber

Due to determination of the chosen models, distributions other than measured can be pictorially represented and in case of necessity tabulated as well (Fig. 5).
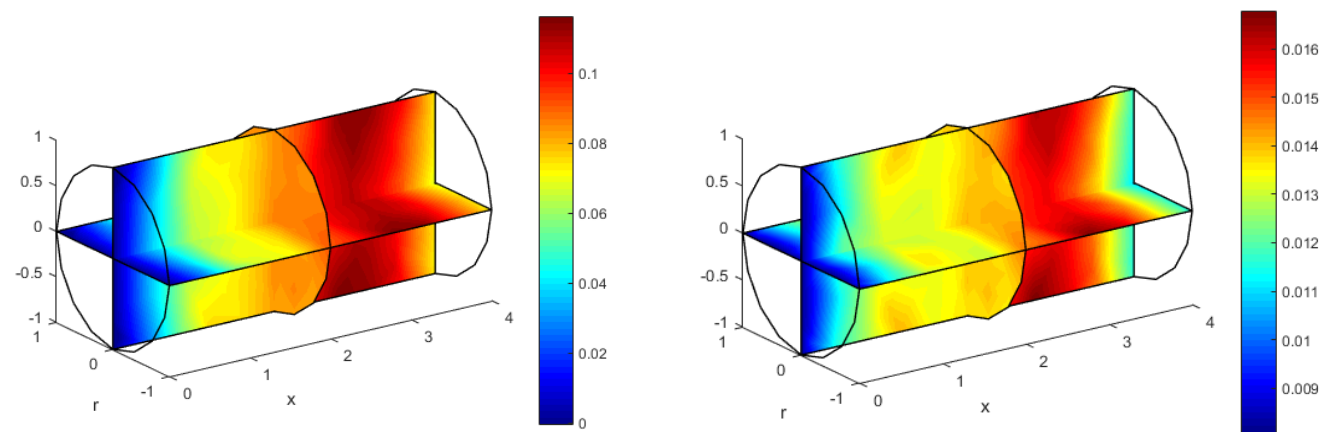

Fig. 5. Distributions of $\mathrm{O}_{2}$ mass fraction (left) and

$\mathrm{H}_{2}$ mass fraction (right) in the combustion chamber

Another advantage of considering PDE solutions is that the information is available at other points than usually measured. The mass fractions are dimensionless in contrast with some of the experimental data. The distributions can and should be made closer to the experimental data depending on parametrization. This research is in progress at the moment.

The brief discussion on usage of different Matlab tools and packages mostly using the finite element method in order to solve $(10,11)$ is presented.

1. PdePE [9]. Good, when considering one spatial and one time dimension. These problems have been considered in $[3 ; 4]$ and have been solved by this package. Hard to compare the fields at each point of the combustion domain. Easy to get the field distributions for post-processing. Is built in standard Matlab installation. In the context of the present experiment would not be enough due to dimensionality, but could be evaluated after certain simplifications of the model, if need be.

2. PdeTools [10]. Powerful, when solving stationary or non-stationary diffusion flame propagation problems, but at first might be tricky to solve problems with variable velocity field. Easy to get the field distributions for post-processing. Not all arrays with the discretization data and data of auxiliary problems of numerical linear algebra are available at least with non-programming approach. Is built in standard Matlab installation. In the context of the present experiment works quite well, since the velocity field considered is constant but due to dimensionality the programming approach has to be applied rather than the built-in interface. 
3. FeaTool [11]. Solves all the previous problems and handles very well more sophisticated ones, such as the ones involving variable velocity fields. Arrays with the discretization data and data of auxiliary problems of numerical linear algebra are partially available for programming approach, but not easy to grasp. Commercial, and the licence has to be obtained independently of the standard Matlab license. In the context of the present experiment works very well and allows to consider more complicated models in the same interface and non-programming approach is enough for the discussed model.

4. Creating own code. No packages required. The main motivation of considering this approach in combination with the ready solutions is the straight-forward usage of arrays containing the discretization data and data of auxiliary problems of numerical linear algebra for post-processing. In the context of the present experiment used several times, when the means were justified and these auxiliary problems were considered.

\section{Conclusions}

1. Different Matlab tools serve for different purposes - depending on the nature of the problem and quite useful, when modelling the considered processes, such as the combustion of different fuels.

2. The choice of the discussed solution obtaining tools can be considered in new related projects naturally appearing today.

3. There is a necessity of introducing the measure between modelling and experimental data - this is something the author is researching at the moment and the framework applicable for present and further experiments is to be obtained.

\section{Acknowledgements}

The author would like to acknowledge the financial support from the ERAF project No. 1.1.1.1/16/A/004.

\section{References}

[1] 2030 climate \& energy framework, Climate Action. [online] [21.03.2021]. Available at: https://ec.europa.eu/clima/policies/strategies/2030_en

[2] National Energy Technology Laboratory (NETL) U. S. Department of Energy. Biomass cofiring program. Program Facts, 2000.

[3] Kalis H., Marinaki M., Strautiņš U., Barmina I. Influence of electric field on thermo-chemical conversion of mixtures of straw pellets with coal, Engineering for Rural Development, 2018, pp. 1746-1753.

[4] Barmina, I., Valdmanis, R., Kalis, H., Marinaki, M. Experimental and numerical study of the development of swirling flow and flame dynamics and combustion characteristics at biomass thermo-chemical conversion, Engineering for Rural Development, 2017, pp. 68-74.

[5] Poinsot T. Veynante D. Theoretical and Numerical Combustion. Edwards, PA USA (2005).

[6] Forman A. Williams. Combustion Theory. The Fundamental Theory of Chemically Reacting Flow Systems. Taylor and Francis, FI USA (1985).

[7] Jay P. Boris Elaine S. Oran. Numerical Simulation of Reactive Flow. Cambridge University Press, NY USA (2001).

[8] Mathworks (2021), URL https://se.mathworks.com/help/matlab/getting-started-with-matlab.html.

[9] Mathworks (2021), [online] [21.03.2021]. Available at: https://se.mathworks.com/help/matlab/ref/pdepe.html

[10] Mathworks (2021), [online] [21.03.2021]. Available at: se.mathworks.com/products/pde.html

[11] FeaTool (2021), [online] [21.03.2021]. Available at: https://www.featool.com/about.

[12]F. M. Rupley R. J. Kee and J. A. Miller. The Chemkin Thermodynamic database. Reaction design, Sandia National Laboratories Report SAND87- 8215B (2000).

[13] Kinetic Mechanism for 20 torr (2004), [online] [21.03.2021]. Available at: http://web.mit.edu /anish/www/pub20torr.mec.

[14] Hall D.L. Torek P.V. Schrock C.R. Donovan M.T. and Wooldridge. H2/O2 reaction mechanism. In: M.S., Proc. Combust. Inst. 29 (2002), in press (2002). 